\title{
TWO COUNTEREXAMPLES IN SEMIGROUP THEORY ON HILBERT SPACE ${ }^{1}$
}

\author{
PAUL R. CHERNOFF
}

\begin{abstract}
There exist $\left(C_{0}\right)$ semigroups $T_{1}(t), T_{2}(t)$ on Hilbert space with the following properties: $T_{1}$ has a bounded generator and is uniformly bounded, but is not similar to a contraction semigroup. $T_{2}$ is uniformly bounded, and there exists no scalar $\alpha$ such that $e^{-\alpha t} T_{2}(t)$ is similar to a contraction semigroup.
\end{abstract}

1. Introduction. If $T(t)=e^{t A}$ is a $\left(C_{0}\right)$ semigroup on a Banach space $X$, then there are real constants $M \geqslant 1$ and $\beta$ such that $\|T(t)\| \leqslant M e^{\beta t}$. If $\beta=0$ the semigroup is said to be uniformly bounded; if, in addition, $M=1$ it is said to be contractive; while if $M=1$ but $\beta \neq 0$ the semigroup is said to be quasicontractive. Clearly $A$ generates a quasi-contractive semigroup if and only if there exists a real $\beta$ such that $A-\beta I$ generates a contractive semigroup, namely $e^{-\beta t} T(t)$. If $T(t)$ is a uniformly bounded semigroup, W. Feller observed that the space $X$ can be renormed to make $T(t)$ contractive; one defines the new norm by $|x|=\sup _{t \geqslant 0}\|T(t) x\|$. Quite generally one can always renorm $X$ by a similar device to make any given $\left(C_{0}\right)$ semigroup quasi-contractive. However, if $X$ is a Hilbert space, the new norm will usually not be a Hilbert norm. Indeed Packel [5] has given an example of a uniformly bounded semigroup $S(t)=e^{t A}$ on Hilbert space $H$ such that there is no equivalent inner product on $H$ which makes $S(t)$ contractive. Equivalently, $S(t)$ is not similar to a contraction semigroup: there is no bounded invertible operator $C$ on $H$ such that $C S(t) C^{-1}$ is a contraction semigroup. The generator $A$ of Packel's semigroup is unbounded, and he asked whether there is an example of such a semigroup with a bounded generator. In $\$ 2$ we shall present such an example. (We note that Kreiss [4] proved that this phenomenon cannot occur in finite dimensions.)

Goldstein [2], [3] has raised a related question: If $T(t)$ is a $\left(C_{0}\right)$ semigroup on Hilbert space $H$, is there an $\alpha$ such that the semigroup $e^{-\alpha t} T(t)$ is similar to a contraction semigroup on $H$ ? In other words, can $H$ be endowed with an equivalent inner product which makes $T(t)$ quasi-contractive? Goldstein's opinion was that the answer is $n o$ in general, and in $\$ 3$ we shall give an example of a semigroup which verifies this conjecture. (The generator of such a semigroup must be unbounded, since if $B$ is bounded we have $\left\|e^{t B}\right\|$ $\leqslant e^{t\|B\|}$, so $e^{t B}$ is quasi-contractive.)

Received by the editors June 25, 1975.

AMS (MOS) subject classifications (1970). Primary 47D05; Secondary 47B44.

${ }^{1}$ Research partially supported by N.S.F. grant GP-30798X. 
2. In this section we exhibit a bounded operator $B$ on a Hilbert space such that the semigroup $e^{t B}$ is uniformly bounded but not similar to a contractive semigroup.

Let $S(t)=e^{t A}$ be Packel's semigroup on the Hilbert space $H$. Then $\|S(t)\| \leqslant M$ for all $t \geqslant 0$ and $S(t)$ is not similar to a contraction semigroup on $H$. For each positive integer $n$ define $A_{n}=A(I-A / n)^{-1}$; then $A_{n}$ is a bounded operator and $\left\|e^{t A_{n}}\right\| \leqslant M$ for $t \geqslant 0$ (cf. the proof of the HilleYosida-Phillips theorem in [1, VIII.1.13]). Let $B_{n}=A_{n} /\left\|A_{n}\right\|$; then $\left\|B_{n}\right\|$ $=1$ and $S_{n}(t)=e^{t B_{n}}$ is uniformly bounded by $M$. Finally, let $\mathcal{C}=\sum \oplus H_{n}$ where each summand $H_{n}$ is a copy of $H$, and let $B=B_{1} \oplus B_{2} \oplus B_{3} \oplus \cdots$ on $\mathcal{H}$. Then $\|B\|=1$ and $\left\|e^{t B}\right\| \leqslant M$ as operators on $\mathcal{H}$.

Proposition. The semigroup $e^{t B}$ is not similar to a contraction semigroup.

Proof. Arguing by contradiction, suppose that $e^{t B}$ is similar to a contraction semigroup. Then there exists an inner product $\langle\cdot, \cdot\rangle$ on $\mathcal{F}$, equivalent to the original inner product $(\cdot, \cdot)$, with respect to which $e^{t B}$ is contractive.

Let $\langle\cdot, \cdot\rangle_{n}$ be the restriction of $\langle\cdot, \cdot\rangle$ to the summand $H_{n}$, which we identify with $H$. Then there is a constant $k>0$ so that

$$
k\langle x, x\rangle_{n} \leqslant(x, x) \leqslant k^{-1}\langle x, x\rangle_{n}
$$

for all vectors $x$ in $H$ and all $n$. Now define a new inner product on $H$ by

$$
[x, y]=\operatorname{LIM}_{n}\langle x, y\rangle_{n}
$$

where LIM is a fixed Banach limit. Then inequality (1) holds for $[x, x]$ as well, so that $[\cdot, \cdot]$ is equivalent to the original inner product on $H$.

Now by assumption $e^{t B_{n}}$ is contractive with respect to the inner product $\langle\cdot, \cdot\rangle_{n}$, hence so is $e^{t A_{n}}$ since $A_{n}$ is just a positive scalar multiple of $B_{n}$. Also, the proof of the Hille-Yosida-Phillips theorem in [1] shows that for all $x$ in $H e^{t A_{n}} x$ converges to $e^{t A} x=S(t) x$. Accordingly,

$$
[S(t) x, S(t) x]=\operatorname{LIM}_{n}\left\langle e^{t A_{n}} x, e^{t A_{n}} x\right\rangle_{n} \leqslant \operatorname{LIM}_{n}\langle x, x\rangle_{n}=[x, x] .
$$

That is, $S(t)$ is contractive with respect to the inner product $[\cdot, \cdot]$, a contradiction.

3. In this section we present an example of a $\left(C_{0}\right)$ semigroup $T(t)$ on Hilbert space such that for no real $\alpha$ is $e^{-\alpha t} T(t)$ similar to a contraction semigroup. The construction makes use of the same machinery employed in $\$ 2$.

As in $\S 2$, let $S(t)$ be Packel's semigroup on $H$, and let $\mathcal{H}$ be the direct sum of countably many copies of $H$. On the space $\mathcal{H}$ define

$$
T(t)=S(t) \oplus S(2 t) \oplus S(3 t) \oplus \cdots .
$$

Proposition. $T(t)$ is a uniformly bounded $\left(C_{0}\right)$ semigroup and there does not exist an $\alpha$ such that $e^{-\alpha t} T(t)$ is similar to a contractive semigroup.

Proof. On the contrary, suppose that for some $\alpha$ there is an equivalent inner product $\langle\cdot, \cdot\rangle$ on $\mathcal{H}$ with respect to which $e^{-\alpha t} T(t)$ is contractive. As in $\S 2$, let $\langle\cdot, \cdot\rangle_{n}$ be the restriction of $\langle\cdot, \cdot\rangle$ to the $n$th summand $H$. Then 
inequalities (1) hold, and we define a new inner product $[\cdot, \cdot]$ on $H$ by a Banach limit (2) as before.

Now $e^{-\alpha t} S(n t)$ is contractive with respect to $\langle\cdot, \cdot\rangle_{n}$. If we replace $t$ by $t / n$ it follows that $e^{-\alpha t / n} S(t)$ is also contractive with respect to $\langle\cdot, \cdot\rangle_{n}$. Applying LIM we deduce that $S(t)$ is contractive with respect to the inner product $[\cdot, \cdot]$, which is again a contradiction.

\section{REFERENCES}

1. N. Dunford and J. T. Schwartz, Linear operators. I:General theory, Pure and Appl. Math., vol. 7, Interscience, New York, 1958. MR 22 \#302.

2. J. A. Goldstein, Contraction semigroups on Hilbert space, Notices Amer. Math. Soc. 21 (1974), A338-A339. Abstract \# 712-B6.

3. _ A problem concerning semigroups on Hilbert space, Tulane Univ.,October, 1974 (preprint).

4. H.-O. Kreiss, Uber Matrizen die beschränkte Halbgruppen erzeugen, Math. Scand. 7 (1959), 71-80.

5. E. W. Packel, A semigroup analogue of Foguel's counterexample, Proc. Amer. Math. Soc. 21 (1969), 240-244. MR 38 \#6400.

Department of Mathematics, University of California, Berkeley, California 94720 